\title{
On hydromagnetic flow of a second-grade fluid induced by an inclined plate
}

Bibhash Deka*, Rita Choudhury

Gauhati University, Department of Mathematics, Guwahati-781014, Assam, India

Corresponding Author Email: bibhashdeka66@gmail.com

https://doi.org/10.18280/ijht.360143

Received: 21 April 2017

Accepted: 10 January 2018

\section{Keywords:}

heat transfer, mass transfer, inclined plate, MHD, visco-elastic, heat generation.

\begin{abstract}
The steady motion of an incompressible electrically conducting laminar visco-elastic fluid over a semi-infinite inclined plate is considered in presence of a magnetic field of uniform strength acting perpendicular to the plate. The dimensionless coupled non-linear differential equations are solved by regular perturbation technique. The enquires are made about the velocity, temperature and concentration fields with shearing stress, Nusselt number and Sherwood number on the wall. Quantitative analysis of the results are presented with a view to disclose the simultaneous effects of heat and mass transfer with the influence of fluid elasticity. It has been shown that the classical solutions are limiting cases of the present analysis. The visco-elastic fluid is found to have immense importance in most polymetric and biological liquids.
\end{abstract}

\section{INTRODUCTION}

Viscoelasticity is the property of fluids that exhibits both viscous and elastic characteristics when undergoing deformation. This type of fluid is widely used in geophysics, chemical engineering, hydrology, biomedical engineering etc. For the last few decades, analysis and modeling of viscoelastic fluid flow dynamics have been the field of very important and active research as this class represents many industrially important fluids like polymers, paints, lubricants, colloidal fluids, suspension fluids etc.

The concept behind the convection flow problems is the density difference caused by simultaneous heat and mass transfer characteristics. In particular, the study of heat and mass transfer with chemical reaction is of

considerable significance in chemical and hydrometallurgical industries. The order of chemical reaction depends on several factors, the simplest of which is the firstorder reaction where the rate of reaction is directly proportional to the species concentration. The study of the heat generation or absorption in moving fluids is important with dissociating fluids. In particular, the effects of heat generation may alter the temperature distribution, correspondingly influences the particle deposition rate in electronic chips, nuclear reactors and semiconductor wafers.

Free-convective Magnetohydrodynamic flow past an inclined plate has exceptional importance in a number of engineering and technological processes. The naturalconvection boundary layer flow over a heated plate with arbitrary inclination is presented by Umemura and Law [1]. Ganesan and Palani [2] have studied the finite difference analysis of unsteady natural convection MHD flow past an inclined plate with variable surface heat and mass flux. Chen [3] has studied the heat and mass transfer in MHD flow with variable wall temperature and concentration. MHD freeconvective flow from an isothermal plate inclined at a small angle to the horizontal is studied by Hossain et al. [4]. Kandasamy and Anjali Devi [5] have presented the effects of chemical reaction, heat and mass transfer along a wedge with heat source and concentration in presence of suction or injection. Turbulent natural-convection between inclined isothermal plates is studied by Said et al. [6]. Sparrow and Husar [7] have presented the longitudinal vortices in natural convection flow on inclined plates. An analytical solution to the problem of radiative heat and mass transfer over an inclined plate at prescribed heat flux with chemical reaction has beeninvestigated by Kumar [8]. Anghel et al. [9] have reported the combined heat and mass transfer by free convection past an inclined flat plate. Kumar [10] has analysed the Heat and mass transfer over an isothermal inclined plate at constant concentration gradient and with heat source.

But it was perceived that most of the industrially important fluids are non-Newtonian. Unlike Newtonian fluids they respond differently under the action of stress imposed on them. In this study, we are interested to analyze a flow problem featured by second-grade fluid which exhibits both viscous and elastic properties. Some recent works are enlisted here. Authors like Choudhury and Das ([11],[12],[13]), Choudhury and Purkayastha [14], Veerakrishna and Reddy ([15],[16]), Acharya et al. [17] etc. have contributed their effects for interpreting the idiosyncrasy of visco-elastic fluid flows.

The objective of this paper is to study the hydromagnetic flow of a second-grade fluid induced by a continuously moving inclined plate in presence of heat and mass transfer. The constitutive equation for the second-grade fluid is taken in the form

$\sigma=-p I+\mu_{1} A_{1}+\mu_{2} A_{2}+\mu_{3} A_{1}^{2}$

where $\sigma$ is the stress tensor, $A_{n}(n=1,2)$ are the kinematic Rivlin-Ericksen tensors. $\mu_{i}(i=1,2,3)$ are the material coefficients describing the viscosity, elasticity and cross viscosity respectively. From thermodynamic considerations, it is noticed that the material coefficients $\mu_{1}$ and $\mu_{3}$ are 
positive and $\mu_{2}$ is negative [Coleman and Markovitz [18]]. The equation (1.1) was deduced by Coleman and Noll [19] from the simple fluids by assuming that stress is more sensitive to the recent deformation than to the deformation that occurred in the distant past. It is reported that solution of poly-isobutylene in cetaneat $30^{\circ} \mathrm{C}$ simulate a second-grade fluid and the material constants for the solutions of various concentrations have been determined by Markovitz.

\section{MATHEMATICAL FORMULATION}

A steady MHD free-convective incompressible flow of a visco-elastic fluid past over a continuously moving semiinfinite inclined plate making an acute angle $\psi$ from the vertical is considered here. The plate is at constant concentration gradient with prescribed mass flux. The flow is in $x$-direction which is taken along the plate and $y$-axis perpendicular to it [Fig 1]. A uniform magnetic field of strength $B_{0}$ is applied normal to the plate. In this study, we assume that the magnetic Reynolds number is very very less than unity so that we can neglect the induced magnetic field in comparison with the applied magnetic field. Also, we assume that all the fluid properties except the density in the buoyancy force term are constant. Then, under the usual Boussinesq's approximation, the governing equations of mass, momentum, energy and concentration are as follows:

$\frac{\partial v}{\partial y}=0$, under the condition $v=$ constant $=-v_{w}, v_{w}>0$.

$v \frac{\partial u}{\partial y}=v_{1} \frac{\partial^{2} u}{\partial y^{2}}+v_{2} v \frac{\partial^{3} u}{\partial y^{3}}+g \beta\left(T-T_{\infty}\right) \cos \psi+g \beta^{\prime}(C-$

$\left.C_{\infty}\right) \cos \psi-\frac{\sigma B_{0}{ }^{2}}{\rho} u$

$v \frac{\partial T}{\partial y}=\frac{k_{t}}{\rho c_{p}} \frac{\partial^{2} T}{\partial y^{2}}+\frac{v_{1}}{c_{p}}\left(\frac{\partial u}{\partial y}\right)^{2}+\frac{v_{2}}{c_{p}} v \frac{\partial u}{\partial y} \frac{\partial^{2} u}{\partial y^{2}}+\frac{Q}{\rho c_{p}}\left(T-T_{\infty}\right)$

$v \frac{\partial C}{\partial y}=D \frac{\partial^{2} C}{\partial y^{2}}-k_{c}\left(C-C_{\infty}\right)$

The corresponding boundary conditions are:

$u=u_{w}, T=T_{w},-D \frac{\partial C}{\partial y}=m_{w}$ at $y=0$

$u \rightarrow 0, T \rightarrow T_{\infty}, C \rightarrow C_{\infty}$ in $y \rightarrow \infty$

For convenience, we introduce the following dimensionless variables:

$Y=\frac{y v_{w}}{v_{1}}, U=\frac{u}{u_{w}}, \theta=\frac{T-T_{\infty}}{T_{w}-T_{\infty}}, \phi=\frac{C-C_{\infty}}{m_{w} v_{1} / v_{w} D}, G r=$ $\frac{g \beta\left(T_{w}-T_{\infty}\right) v_{1}}{u_{w} v_{w}^{2}}, G c=\frac{g \beta^{\prime} m_{w} v_{1}^{2}}{u_{w} v_{w}{ }^{3} D}$,

$M=\frac{B_{0}^{2} v_{1} \sigma}{v_{w}^{2} \rho}, \operatorname{Pr}=\frac{\rho v_{1} c_{p}}{k_{t}}, K c=\frac{v_{1} k_{c}}{v_{w}{ }^{2}}, S c=\frac{v_{1}}{D}, E=$

$\frac{u_{w}{ }^{2}}{\left(T_{w}-T_{\infty}\right) c_{p}}, S=\frac{Q v_{1}}{\rho c_{p} v_{w}{ }^{2}}, \alpha_{1}=\frac{v_{w}{ }^{2} v_{2}}{v_{1}^{2}}$

Substitution of (2.7) into the equations (2.2) to (2.4) yields the following dimensionless equations:

$\frac{d^{2} U}{d Y^{2}}+\frac{d U}{d Y}-\alpha_{1} \frac{d^{3} U}{d Y^{3}}=M U-G r \theta \cos \psi-G c \phi \cos \psi$ $\frac{d^{2} \theta}{d Y^{2}}+\operatorname{Pr} \frac{d \theta}{d Y}=\operatorname{Pr} E\left\{\alpha_{1} \frac{d U}{d Y} \frac{d^{2} U}{d Y^{2}}-\left(\frac{d U}{d Y}\right)^{2}\right\}-\operatorname{Pr} S \theta$

$\frac{d^{2} \phi}{d Y^{2}}+S c \frac{d \phi}{d Y}=S c K c \phi$

with relevant boundary conditions:

$U=1, \theta=1, \frac{\partial \phi}{\partial Y}=-1$ at $Y=0$

$U \rightarrow 0, \theta \rightarrow 0, \phi \rightarrow 0$ in $Y \rightarrow \infty$

\section{ANALYTICAL SOLUTION OF THE PROBLEM}

The solution of equation (2.10) is given by

$\phi=\frac{2}{S c+\sqrt{S c^{2}+4 . K c \cdot S c}} e^{-A_{3} Y}$

To solve the differential equations (2.8) and (2.9) under subject to the boundary conditions (2.11) and (2.12), we use perturbation technique and take the Eckert number $E$ as perturbation parameter as $E$ is very small for incompressible fluid. Expanding $U$ and $\theta$ in powers of the Eckert number $E$ and neglecting the higher order terms of $o\left(E^{2}\right)$, we have

$U=U_{0}+E U_{1}+o\left(E^{2}\right)$

$\theta=\theta_{0}+E \theta_{1}+o\left(E^{2}\right)$

Substituting (3.2) and (3.3) in the equations (2.8) and (2.9) and equating like powers of $E$, we get the following equations:

Zeroth-order equations:

$U_{0}{ }^{\prime \prime}+U_{0}{ }^{\prime}-\alpha_{1} U_{0}{ }^{\prime \prime \prime}=M U_{0}-G r \theta_{0} \cos \psi-G c \phi \cos \psi$

$\theta_{0}^{\prime \prime}+\operatorname{Pr} \theta_{0}^{\prime}+\operatorname{PrS} \theta_{0}=0$

First- order equations:

$U_{1}^{\prime \prime}+U_{1}{ }^{\prime}-\alpha_{1} U_{1}{ }^{\prime \prime \prime}=M U_{1}-G r \theta_{1} \cos \psi$

$\theta_{1}^{\prime \prime}+\operatorname{Pr} \theta_{1}{ }^{\prime}+\operatorname{Pr} S \theta_{1}=\operatorname{Pr}\left(\alpha_{1} U_{0}{ }^{\prime} U_{0}{ }^{\prime \prime}-U_{0}{ }^{2}\right)$

subject to the boundary conditions:

$U_{0}=1, U_{1}=0 ; \theta_{0}=1, \theta_{1}=0$ at $Y=0$

$U_{0}=0=U_{1} ; \theta_{0}=0=\theta_{1}$ in $Y \rightarrow \infty$

The solution of (3.5) subject to the boundary conditions (3.8) and (3.9) gives

$\theta_{0}=e^{-A_{2} Y}$

To solve coupled differential equations (3.4), (3.6) and (3.7), we use multi parameter perturbation technique following Nowinski and Ismail [20] as $\alpha_{1} \ll 1$ for small shear rate. Expanding $U_{0}$ and $U_{1}$ in powers of the viscoelastic parameter $\alpha_{1}$ and neglecting the higher -order terms of $o\left(\alpha_{1}^{2}\right)$, we have 
$U_{0}=U_{00}+\alpha_{1} U_{01}+o\left(\alpha_{1}^{2}\right)$

$U_{1}=U_{10}+\alpha_{1} U_{11}+o\left(\alpha_{1}^{2}\right)$

Now, substituting (3.11) and (3.12) in the differential equations (3.4) and (3.7) and equating the like powers of $\alpha_{1}$, we get the following equations:

Zeroth-order equations:

$U_{00}{ }^{\prime \prime}+U_{00}{ }^{\prime}-M U_{00}=-G r \theta_{0} \cos \psi-G c \phi \cos \psi$

$U_{10}{ }^{\prime \prime}+U_{10}{ }^{\prime}-M U_{10}=-b_{25} e^{-A_{2} Y}+b_{26} e^{-2 A_{1} Y}+$ $b_{27} e^{-2 A_{2} Y}+b_{28} e^{-2 A_{3} Y}-b_{29} e^{-\left(A_{1}+A_{2}\right) Y}-b_{30} e^{-\left(A_{1}+A_{3}\right) Y}+$ $b_{31} e^{-\left(A_{3}+A_{2}\right) Y}$

First-order equations:

$U_{01}{ }^{\prime \prime}+U_{01}{ }^{\prime}-M U_{01}=U_{00}{ }^{\prime \prime \prime}$

$U_{11}{ }^{\prime \prime}+U_{11}{ }^{\prime}-M U_{11}=U_{10}{ }^{\prime \prime \prime}-b_{41} e^{-A_{2} Y}-\left(b_{32}+b_{38}-\right.$ $\left.b_{12} G r Y \cos \psi\right) e^{-2 A_{1} Y}-b_{33} e^{-2 A_{2} Y}-b_{34} e^{-2 A_{3} Y}-$ $\left(b_{35}-b_{39}+b_{13} G r Y \cos \psi\right) e^{-\left(A_{1}+A_{2}\right) Y}-\left(b_{36}-b_{40}+\right.$ $\left.b_{14} G r Y \cos \psi\right) e^{-\left(A_{1}+A_{3}\right) Y}-b_{37} e^{-\left(A_{3}+A_{2}\right) Y}$

with relevant boundary conditions:

$U_{00}=1, U_{01}=0, U_{10}=0, U_{11}=0$ at $Y=0$

$U_{00} \rightarrow 0, U_{01} \rightarrow 0, U_{10} \rightarrow 0, U_{11} \rightarrow 0$ in $Y \rightarrow \infty$

Solving the system of equations (3.13) to (3.16), using boundary conditions (3.17) and (3.18), weget the expressions for $U_{00}, U_{01}, U_{10}$ and $U_{11}$ and consequently the expressions for $U_{0}$ and $U_{1}$. Now, using the value of $U_{0}$ in (3.7) and after solving this equation, we get the expression for $\theta_{1}$. Finally, substitution of these values in (3.2) and (3.3) yields the velocity and temperature of the problem as follows:

$U=b_{5} e^{-A_{1} Y}-b_{1} e^{-A_{2} Y}-b_{3} e^{-A_{3} Y}+\alpha_{1}\left\{-\left(b_{3}+\right.\right.$ $\left.\left.b_{4}\right) e^{-A_{1} Y}+\frac{b_{5}}{\sqrt{1+4 M}} A_{1}{ }^{3} Y e^{-A_{1} Y}+b_{3} e^{-A_{2} Y}+b_{4} e^{-A_{3} Y}\right\}+$ $E\left[\left\{b_{49} e^{-A_{1} Y}-b_{42} e^{-A_{2} Y}+b_{43} e^{-2 A_{1} Y}+b_{44} e^{-2 A_{2} Y}+\right.\right.$ $b_{45} e^{-2 A_{3} Y}-b_{46} e^{-\left(A_{1}+A_{2}\right) Y}-b_{47} e^{-\left(A_{1}+A_{3}\right) Y}+$

$\left.b_{48} e^{-\left(A_{3}+A_{2}\right) Y}\right\}+\alpha_{1}\left\{\left(b_{66}-b_{65} Y\right) e^{-A_{1} Y}+b_{52} e^{-A_{2} Y}-\right.$ $\left(b_{53}+b_{64}-b_{63} Y\right) e^{-2 A_{1} Y}-b_{54} e^{-2 A_{2} Y}-b_{55} e^{-2 A_{3} Y}+$ $\left(b_{56}+b_{51}-b_{50} Y\right) e^{-\left(A_{1}+A_{2}\right) Y}+\left(b_{57}+b_{62}-\right.$ $\left.\left.\left.b_{61} Y\right) e^{-\left(A_{1}+A_{3}\right) Y}-b_{58} e^{-\left(A_{3}+A_{2}\right) Y}\right\}\right]$

$\theta=e^{-A_{2} Y}+E\left\{b_{24} e^{-A_{2} Y}+\left(b_{15}+b_{21}-b_{12} \alpha_{1} Y\right) e^{-2 A_{1} Y}+\right.$ $b_{16} e^{-2 A_{2} Y}+b_{17} e^{-2 A_{3} Y}+\left(b_{18}-b_{22}+\right.$ $\left.b_{13} \alpha_{1} Y\right) e^{-\left(A_{1}+A_{2}\right) Y}+\left(b_{19}-b_{23}+b_{14} \alpha_{1} Y\right) e^{-\left(A_{1}+A_{3}\right) Y}+$ $\left.b_{20} e^{-\left(A_{3}+A_{2}\right) Y}\right\}$

The constants are not presented here due to sake of brevity.

\section{RESULTS AND DISCUSSION}

Knowing the velocity, temperature and concentration fields, we discuss some important flow characteristics of the problem viz. wall shear stress, local heat flux and mass flux.
The non-dimensional shearing stress $\tau$ at the plate $Y=0$ is given by

$\left.\tau=\frac{d U}{d Y}-\alpha_{1} \frac{d^{2} U}{d Y^{2}}\right]_{Y=0}=b_{67}+b_{68}+b_{69}-b_{70}$

The non-dimensional heat flux at the plate $Y=0$ in terms of Nusselt number $\mathrm{Nu}$ is given as

$$
\begin{aligned}
& \left.N u=\frac{d \theta}{d Y}\right]_{Y=0}=-A_{2}+E\left\{-A_{2} b_{24}-2 A_{1} b_{15}-2 A_{2} b_{16}-\right. \\
& 2 A_{3} b_{17}-b_{18}\left(A_{1}+A_{2}\right)-b_{19}\left(A_{1}+A_{3}\right)-b_{20}\left(A_{2}+A_{3}\right)- \\
& 2 A_{1} b_{21}+b_{22}\left(A_{2}+A_{1}\right)+b_{23}\left(A_{1}+A_{3}\right)-\alpha_{1}\left(b_{12}-b_{13}-\right. \\
& \left.\left.b_{14}\right)\right\}
\end{aligned}
$$

The non-dimensional mass flux at the plate $Y=0$ in terms of Sherwood number $S h$ is expressed by

$\left.S h=\frac{d \phi}{d Y}\right]_{Y=0}=-1$

In order to get an insight into the physical situation of the problem, the numerical computations have been carried out for velocity, temperature and concentration fields. Throughout the computations, we employ different values of the visco-elastic parameter $\alpha_{1}$, thermal Grashof number $G r$, solutal Grashof number $G c$, magnetic parameter M, Prandtl number $\mathrm{Pr}$, chemical reaction parameter $K c$, heat source parameter $S$ with fixed value of Eckert number $\mathrm{E}=.01$, Schmidt number $S c=3$ and angle of inclination $\psi=\frac{\pi}{4}$. The non-zero values of $\alpha_{1}$ characterize the visco-elastic fluid and $\alpha_{1}=0$ represents the character of Newtonian fluid flow phenomenon.

Figures 2 to 8 demonstrate the variation of fluid velocity $U$ against $Y$ with various values of other flow parameters. The graphs reveal that the fluid velocity gradually diminishes away from the plate for both Newtonian and visco-elastic fluids. Also, with the growth of the absolute values of the visco-elastic parameter $\alpha_{1}\left(\alpha_{1}=0,-0.1,-0.2\right)$ the fluid velocity decelerates in comparison with Newtonian fluid flow phenomenon.

In figures 2 and 3, it is observed that the convection velocity follows a downward trend in the case of cooling plate i.e. $G r>0$ in comparison with the heated plate i.e. $G r<0$ in both Newtonian and non-Newtonian cases. The solutal Grashof number $G c$ is the ratio of species buoyancy force to the viscous force in the boundary layer flow and $G c>0$ infers that the free stream concentration is less than the concentration at the boundary surface. With the enhancement of $G c$, species buoyancy force increases and as a result the fluid velocity shows an increasing trend in both Newtonian and non-Newtonian cases (Figs. 2 and 4). An increase in magnetic parameter $M$ diminishes the fluid velocity (Figs. 2 and 5). The magnetic parameter is a consequence of Lorentz force which is a frictional force acts in the opposite direction of fluid motion. Thus, with the increase in Lorentz forcethe velocity decreases in both Newtonian as well as non-Newtonian fluids. The rising values of the chemical reaction parameter $K c$ (Figs. 2 and 7) and the heat source parameter $S$ (Figs. 2 and 8) depict a decelerating trend of fluid velocity but an opposite behaviour in fluid velocity is experienced during the growing behaviour of Prandtl number $\operatorname{Pr}$ (Figs. 2 and 6). 
From practical point of view, we cannot ignore the effects of visco-elastic parameter on the resistive force or viscous drag. The shearing stress is one kind of resistive force or viscous drag on the surface of a body due to the motion of the fluid. The variationsof shearing stress against the thermal Grashofnumber $\mathrm{Gr}$, the solutal Grashof number $G c$, the magnetic parameter $M$, the Prandtl number $\mathrm{Pr}$, the chemical reaction parameter $K c$ and heat source parameter $S$ are represented by the figures 9 to 14 respectively with fixed values of other physical parameters as mentioned. The growth of $G r, G c$ and $S$ enhance the viscous drag formed by Newtonian and visco-elastic fluids at the plate but an opposite trend is noticedin case of the parameters $M, P r$ and $K c$ in both the fluid flow systems. Again, it is observed that the shearing stress enhances with the growth of absolute value of $\alpha_{1}$ against indicated flow parameters for both types of fluids.

The graphical presentation of the temperature $\theta$ against $Y$ is given inthe figure 15. From the figure, it has been observed that the temperature is not significantly affected by the viscoelastic parameter. The similar conclusion is true for Nusselt number $\mathrm{Nu}$.

\section{CONCLUSIONS}

The study leads to the following conclusions:

- The velocity field is significantly affected at each point of the fluid flow region by the visco-elastic parameter.

- The growth of the absolute value of the viscoelasticity depicts the decelerating trend of fluid velocity in comparison with Newtonian fluid flow phenomenon.

- With the enhancement of different significant flow parameters the shearing stress affects considerably in both Newtonian and visco-elastic cases.

- The temperature profile is not considerably affected by the visco-elastic parameter. This is due to the restraining effect played by the elasticity of the fluid.

- The Nusselt number and Sherwood number are not significantly affected by the visco-elastic parameter.

\section{SCOPE OF THE WORK}

The above outcomes excel to conclude that though this study is analytical in nature but it has many eloquent applications in diversified fields of engineering and technology.

\section{FIGURES}

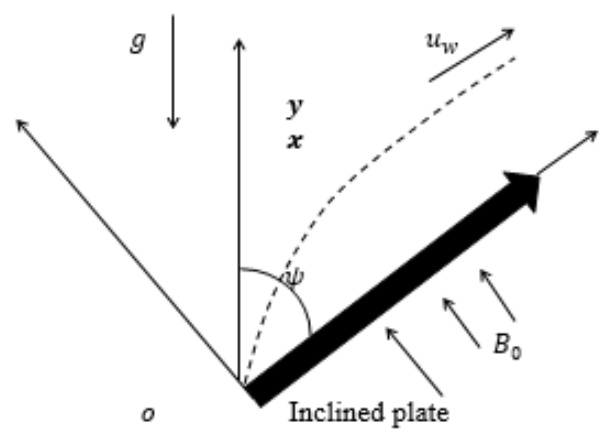

Figure 1. Physical model and co-ordinate system.

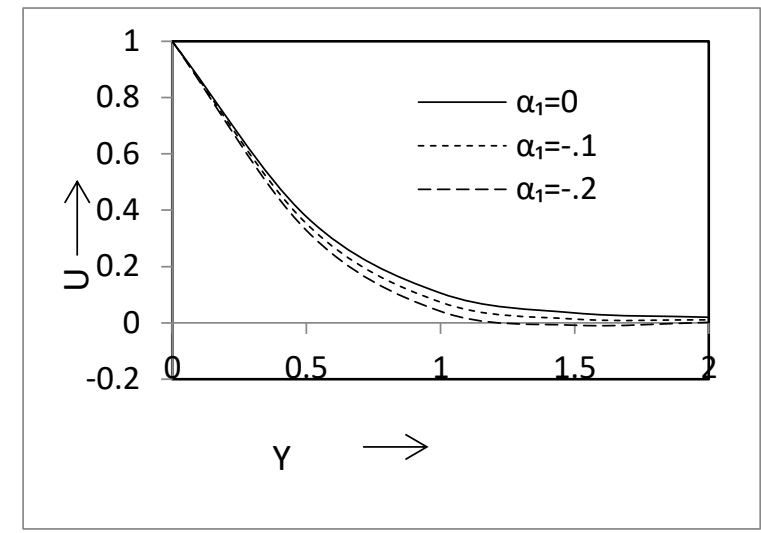

Figure 2. Variation of $U$ against $Y$ for $G r=2, G c=6, M=$ $2, \operatorname{Pr}=3, K c=4, S=3.5$

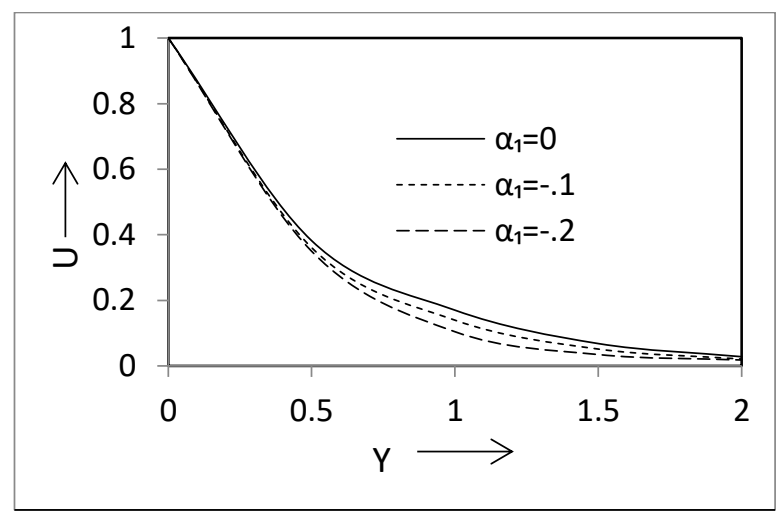

Figure 3. Variation of $U$ against $Y$ for $G r=-2, G c=6$, $M=2, \operatorname{Pr}=3, K c=4, S=3.5$.

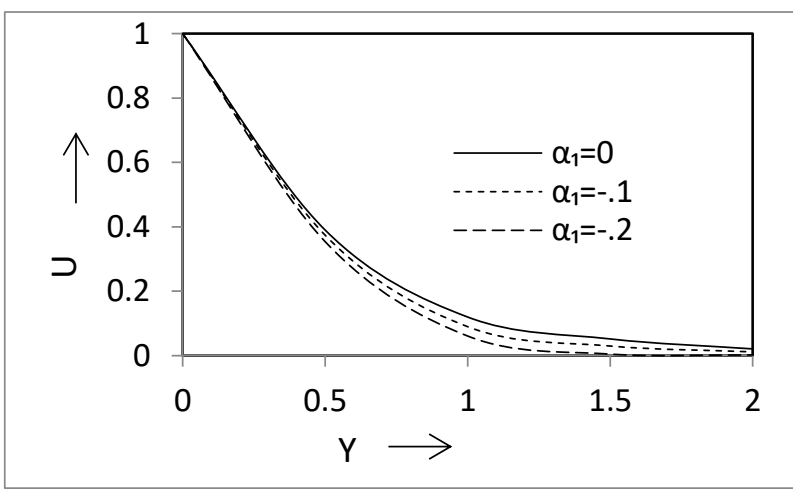

Figure 4. Variation of $U$ against $Y$ for $G r=2, G c=10$, $M=2, \operatorname{Pr}=3, K c=4, S=3.5$.

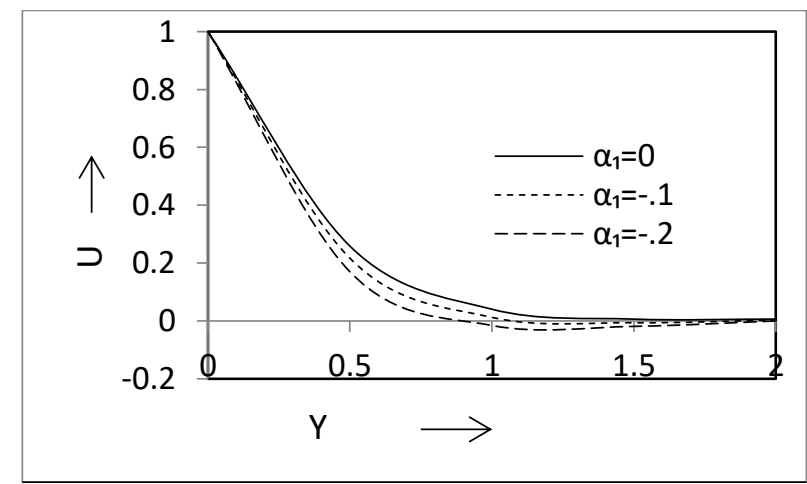

Figure 5. Variation of $U$ against $Y$ for $G r=2, G c=6$, $M=5, P r=3, K c=4, S=3.5$. 


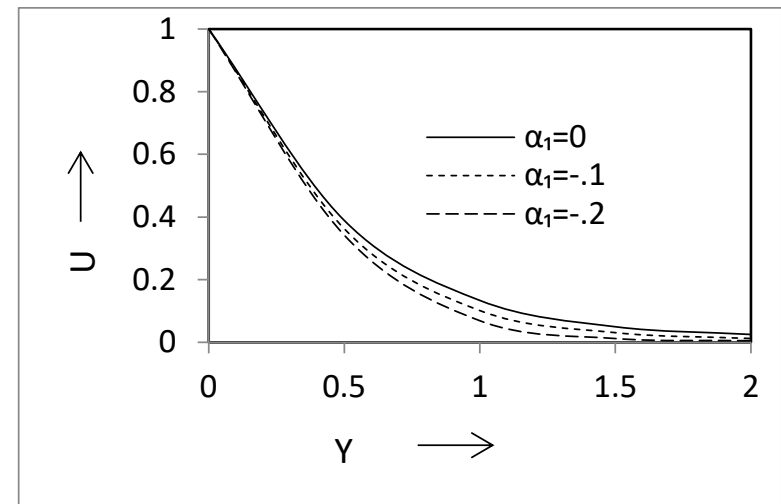

Figure 6. Variation of $U$ against $Y$ for $G r=2, G c=6, M=$ $2, P r=6, K c=4, S=3.5$.

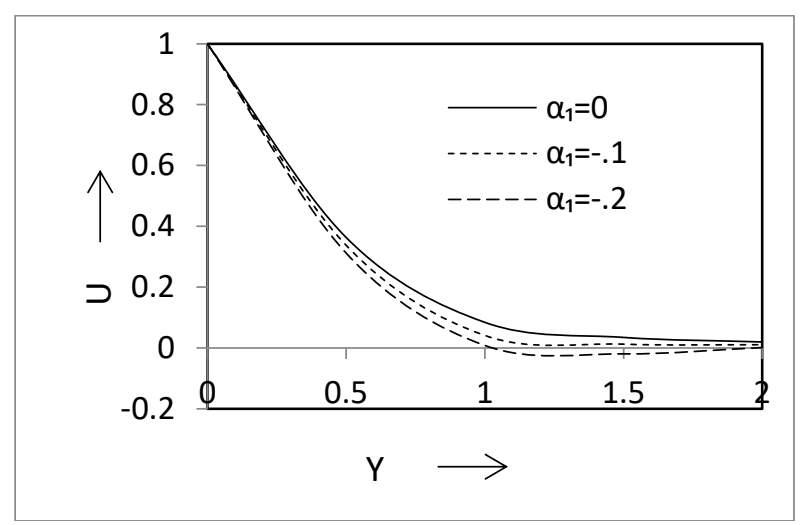

Figure 7. Variation of $U$ against $Y$ for $G r=2, G c=6, M=$ $2, \operatorname{Pr}=3, K c=6, S=3.5$.

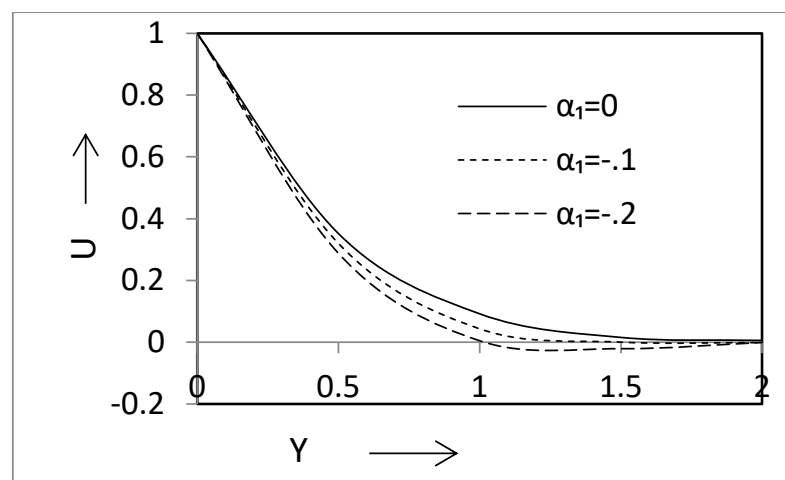

Figure 8. Variation of $U$ against $Y$ for $G r=2, G c=6, M=$ $2, \operatorname{Pr}=3, K c=4, S=7$.

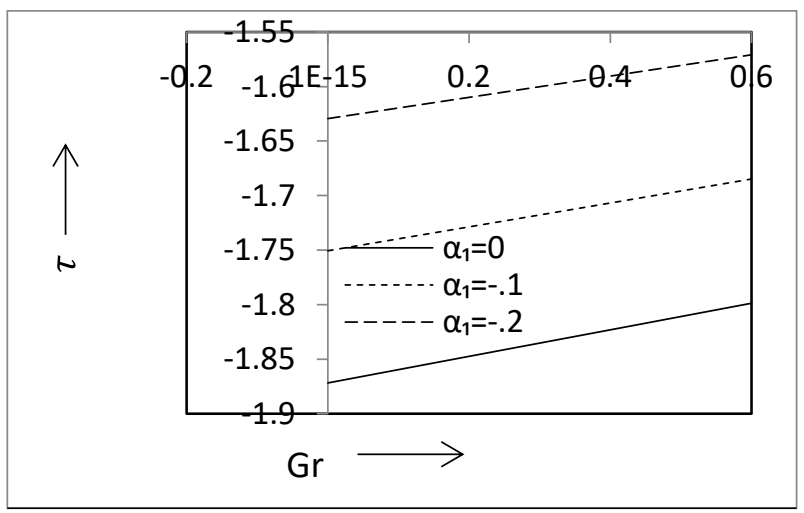

Figure 9. Variation of $\tau$ against $G r$ for $G c=6, M=2, P r=$ $3, K c=4, S=3.5$.

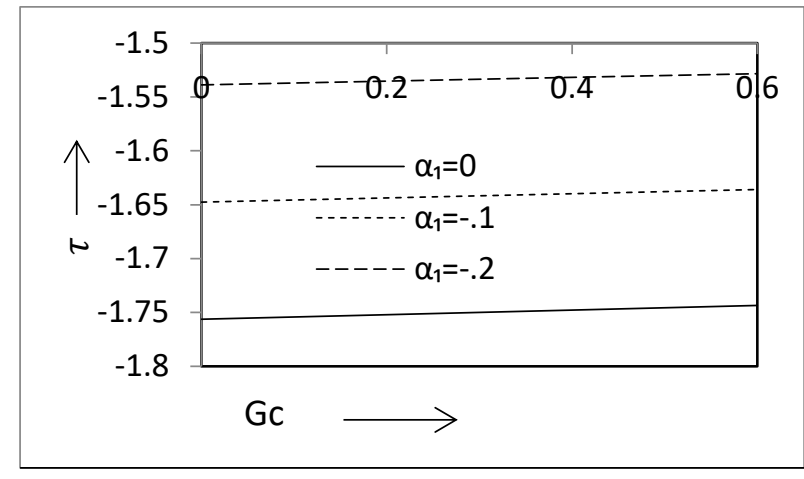

Figure 10. Variation of $\tau$ against $G c$ for $G r=2, M=$ $2, \operatorname{Pr}=3, K c=4, S=3.5$.

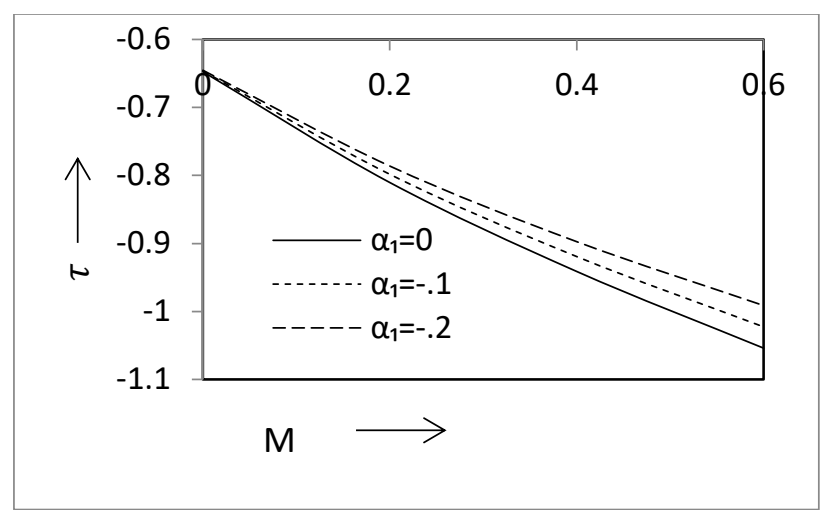

Figure 11. Variation of $\tau$ against $M$ for $G r=2, G c=$ $6, \operatorname{Pr}=3, K c=4, S=3.5$.

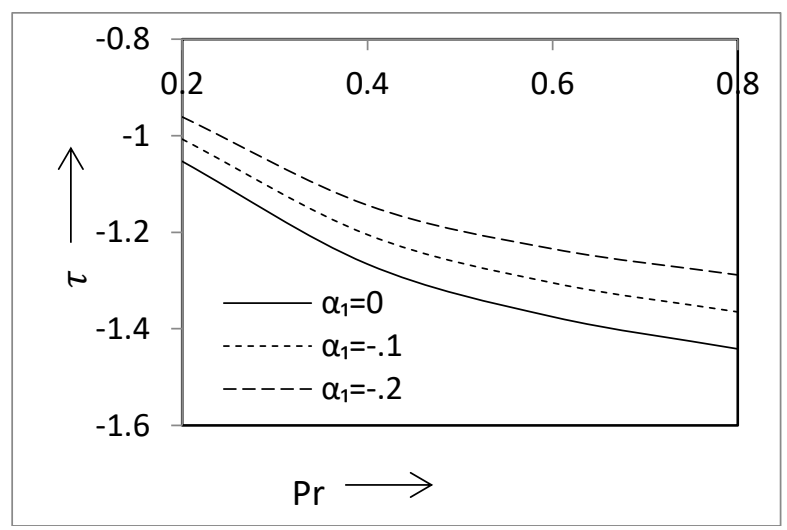

Figure 12. Variation of $\tau$ against $\operatorname{Pr}$ for $G r=2, G c=$ $6, M=2, K c=4, S=3.5$.

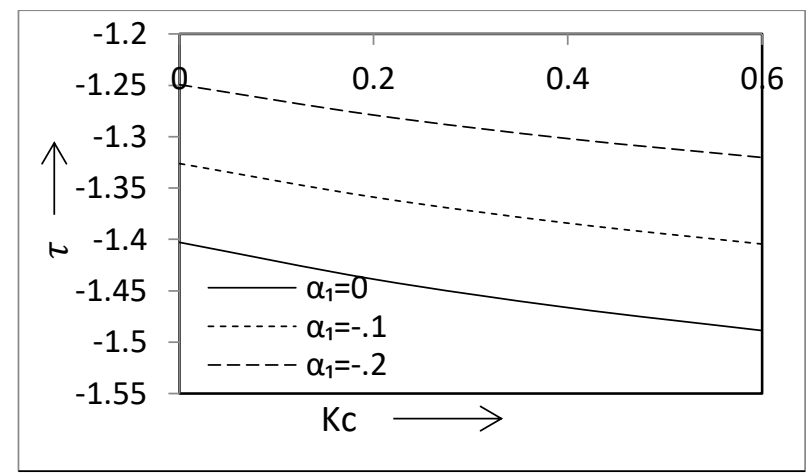

Figure 13. Variation of $\tau$ against $K c$ for $G r=2, G c=$ 6, $M=2, \operatorname{Pr}=3, S=3.5$ 


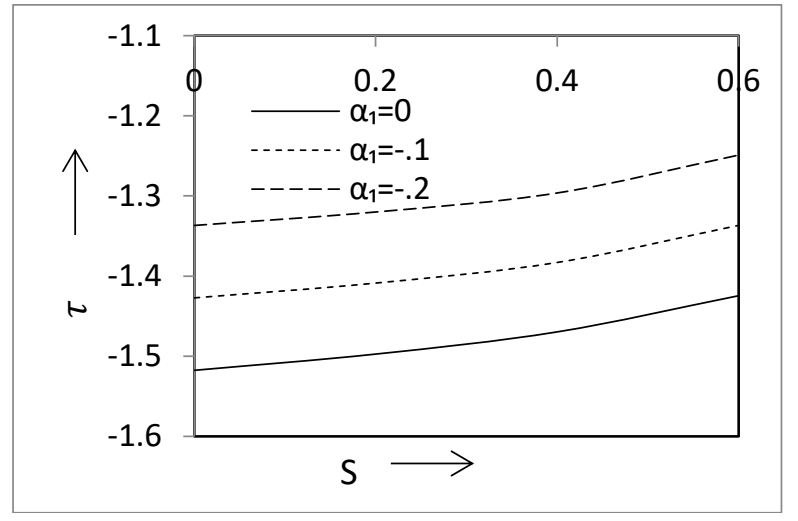

Figure 14. Variation of $\tau$ against $S$ for $G r=2, G c=6, M=$ $2, \operatorname{Pr}=3, K c=4$.

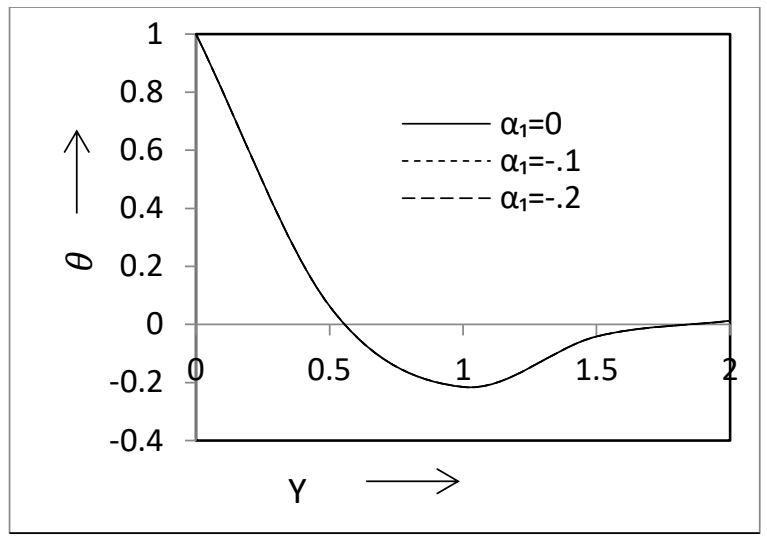

Figure 15. Variation of $\theta$ against $Y$ for $G r=2, G c=6$, $M=2, \operatorname{Pr}=3, K c=4, S=3.5$.

\section{ACKNOWLEDGMENT}

The financial support by UGC is gratefully acknowledged by the authors.

\section{REFERENCES}

[1] Umemura A, Law CK. (1990). Natural convection boundary layer flow over a heated plate with arbitrary inclination. J. Fluid Mech. 219: 571-584.

[2] Ganesan P, Palani G. (2004). Finite difference analysis of unsteady natural-convection MHD flow past an inclined plate with variable surface heat and mass flux. Int. J. Heat Mass transfer. 47: 4449-4457.

[3] Chen CH. (2004). Heat and mass transfer in MHD flow with variable wall temperature and Concentration. Acta Mech. 172: 219-235.

[4] Hossain MA, Pop I, Ahmad M. (1996). MHD freeconvective flow from an isothermal plate inclined at a small angle to the horizontal, J. Theo. Appl. Fluid Mech. 1: 194- 207.

[5] Kandasamy R, Anjali Devi SP. (2004). Effects of chemical reaction, heat and mass transfer along a wedge with heat source and concentration in presence of suction or injection. J. of comput. Appl. Mech. 5: 21-31.

[6] Said SAM, Habib MA, Badr HM, Anwar S. (2005). Turbulent natural-convection between inclined isothermal plates. Computers and Fluid. 34: 1025-1039.
[7] Sparrow EM, Husar RB. (1969). Longitudinal vortices in natural-convection flow on inclined Plates. J. Fluid. Mech. 37: 251-255.

[8] Kumar H. (2012), An analytical solution to the problem of radiative heat and mass transfer over an inclined plate at prescribed heat flux with chemical reaction. J. Serb. Chem. Soc. 77: 1-14.

[9] Hossain AM, Zeb MA, Pop SI. (2001). Combined heat and mass transfer by free- convection past an inclined flat plate. Int. J. Appl. Mech. Engg. 2: 219-235.

[10] Kumar H. (2013). Heat and mass transfer over an isothermal inclined plate at constant concentration gradient and with heat source. World App. Sci. J. 24: 364-369.

[11] Choudhury R, Das UJ. (2012). MHD mixed convective heat and mass transfer in a visco-elastic boundary layer slip flow past a vertical permeable plate with thermal radiation and chemical reaction. Int. J. Statistika and Mathematika 3: 93-101.

[12] Choudhury R, Das UJ. (2013). Visco-elastic effects on three dimensional hydromagnetic flow past a vertical porous plate. Int. J of Heat and Tech. 31: 1-8.

[13] Choudhury R, Das B. (2016). Influence of viscoelasticity on MHD heat and mass transfer flow through a porous medium bounded by an inclined surface with chemical reaction. Int. J. of Heat and Tech. 34: 332-338.

[14] Choudhury R, Purkayastha S. (2014). An analysis of mixed convective elastic-viscous fluid past a vertical porous plate in presence of induced magnetic field and chemical reaction. Int. J. of Heat and Tech. 32: 27-34.

[15] Veerakrishna M, Reddy MG. (2016). MHD freeconvective rotating flow of visco-elastic fluid past an infinite vertical oscillating porous plate with chemical reaction. IOP Conf. Series: Material Sci. and Engg. 149

[16] Veerakrishna M, Reddy GS. (2016). Unsteady MHD convective flow of a second-grade fluid through a porous medium in a rotating parallel plate channel with temperature dependent source. IOP Conf. Series: Material Sci. and Engg. 149

[17] Acharya N, Das K, Kundu PK. (2017). Outlining the impact of second-order slip and multiple convective condition on nano fluid flow: a new statistical layout. Can. J. Phys.: 1-8.

[18] Coleman BD, Markovitz H. (1964). Incompressible second-order fluids. Adv. Appl. Mech. 8: 69-101.

[19] Coleman BD, Noll W. (1960). An applications theorem for functional with applications in continuum mechanics. Archs Ration Mech. Analysis 6: 350-360.

[20] Nowinski SK, Ismail IA. (1965). Application of multiparameter perturbation methods to elasto-statics in development of theoretical and applied mechanics. Pergamon Press.

\section{NOMENCLATURE}

$u$ and $v$ are velocity components along $x$ and $y$ axes respectively, $u_{w}$ is the plate velocity, $v_{w}$ is the suction velocity, $v_{1}$ is the kinematic viscosity, $v_{2}$ is the viscoelasticity, $g$ is the acceleration due to gravity, $\beta$ is the coefficient of volumetric expansion for heat transfer, $\beta^{\prime}$ is the coefficient of volumetric expansion for mass transfer, $T$ is the fluid temperature, $T_{w}$ is the temperature of the plate, $T_{\infty}$ is the temperature of the fluid far away from the plate, $C$ is the 
species concentration, $C_{\infty}$ is the species concentration of the fluid far away from the plate, $\sigma$ is the electrical conductivity, $\rho$ is the density of the fluid, $k_{t}$ is the thermal conductivity, $c_{p}$ is the specific heat at constant pressure, $Q$ is the heat generation coefficient, $D$ is the mass diffusion coefficient, $k_{c}$ is the rate of chemical reaction, $m_{w}$ is the plate mass flux, $Y$ is the non-dimensional horizontal coordinate, $U$ is the dimensionless axial velocity, $\theta$ is the dimensionless temperature, $\phi$ is the non-dimensional species concentration, $\mathrm{Pr}$ is the Prandtl number, $S c$ is the Schmidt number, $M$ is the Hartmann number, $G r$ is the thermal Grashof number, $G c$ is the solutal Grashof number, $\mathrm{E}$ is the Eckert number, $K c$ is the chemical reaction parameter, $S$ is the heat source parameter and $\alpha_{1}$ is the visco-elastic parameter. 